University of Nebraska - Lincoln

DigitalCommons@University of Nebraska - Lincoln

February 2002

\title{
THE INTERMEDIATE-PERIOD CEPHEID STRIP STARS
}

Edward G. Schmidt

University of Nebraska-Lincoln, eschmidt1@unl.edu

Follow this and additional works at: https://digitalcommons.unl.edu/physicsschmidt

Part of the Physics Commons

Schmidt, Edward G., "THE INTERMEDIATE-PERIOD CEPHEID STRIP STARS" (2002). Edward Schmidt Publications. 3.

https://digitalcommons.unl.edu/physicsschmidt/3

This Article is brought to you for free and open access by the Research Papers in Physics and Astronomy at DigitalCommons@University of Nebraska - Lincoln. It has been accepted for inclusion in Edward Schmidt Publications by an authorized administrator of DigitalCommons@University of Nebraska - Lincoln. 


\title{
THE INTERMEDIATE-PERIOD CEPHEID STRIP STARS
}

\author{
EDWARD G. SCHMIDT \\ Department of Physics and Astronomy, University of Nebraska, Lincoln, NE 68588; eschmidt1@unl.edu \\ Received 2001 September 13; accepted 2001 October 29
}

\begin{abstract}
To explore the population of variable stars that fall between RR Lyrae stars and classical Cepheids, we have obtained extensive photometric observations for 56 stars with periods between 0.6 and 1.0 days. For these stars and an additional 10, we have examined the form and stability of the light curves to explore the variety of types of stars in this period regime. We conclude that the majority of the sample are Bailey type ab RR Lyrae stars. However, a surprising number that possibly exhibit the Blazhko effect are found. There are a half-dozen other stars that do not appear to be in either of these two groups. One is the well-known multimode pulsator AC And. We suggest that two of the others may be anomalous Cepheids and two may be type II Cepheids.
\end{abstract}

Key words: Cepheids — RR Lyrae variable

On-line material: machine-readable table

\section{INTRODUCTION}

The two most numerous classes of stars in the Cepheid instability strip, RR Lyrae stars and classical Cepheids, have been extensively studied. It can be argued that our understanding of these objects is now quite complete. On the other hand, there is considerable uncertainty surrounding the nature and properties of other instability strip stars. This is clearly the case for stars with periods between the longest period RR Lyrae stars and the shortest period Cepheids. We shall refer to these as intermediate-period Cepheid strip stars. In light of the discussion below and to be sufficiently inclusive, the period range will be taken to be from 0.6 to 3 days.

The General Catalog of Variable Stars (GCVS; Kholopov 1985, 1987) lists only about 960 pulsating stars with periods between 0.6 days and 1 day compared with more than 5600 at shorter periods. Similarly, the GCVS contains about 44 pulsating stars in the range from 1 to 2.5 days compared with more than 650 Cepheid strip stars with longer periods. This relatively small population in the intermediate region simply represents the frequency minimum between two numerous classes of stars. It is also no doubt exaggerated by selection effects against the discovery of stars with periods near 1 day.

The situation with respect to globular cluster stars is somewhat more complex. In intermediate-metallicity, Oosterhoff I clusters, less than $20 \%$ of the Bailey type ab RR Lyrae stars have periods longer than 0.6 days (Kukarkin 1975), a similar proportion to that among field stars. On the other hand, in metal-poor, Oosterhoff II clusters, the proportion often exceeds $60 \%$. The differences between field and cluster stars is a long-standing issue that needs to be addressed.

In addition to RR Lyrae stars and classical Cepheids, it has been suggested that this regime may contain anomalous Cepheids (intermediate-period, Population II variables that are overluminous and probably overmassive, possibly as the result of stellar mergers; Nemec 1989), overtone type II Cepheids (post-horizontal-branch stars; Teays \& Simon 1985), first- and second-overtone classical Cepheids (evolved Population I stars; Alcock et al. 1995), and three types of AHB variables (stars evolving away from the horizontal branch toward the asymptotic giant branch; Diethelm 1990). Layden et al. (1999) have also proposed a subclass of metal-rich RR Lyrae stars with periods between 0.5 and 0.9 days. To add to the confusion, some of these groups overlap, so that a given star might legitimately be a member of more than one of them.

The identification of individual stars with a particular class (i.e., evolutionary history) is sometimes difficult. Thus, Simon \& Teays (1982) argued that the sinusoidal light curve of XZ Cet $(P=0.82$ days) might be explained by a period resonance among long-period RR Lyrae but later found this unlikely in light of pulsational calculations (Teays \& Simon 1985). They were then unable to choose definitively between the possibilities of its being an overtone type II Cepheid or an anomalous Cepheid. Similarly, Schmidt et al. (1990) found evidence from the Fourier parameters of the light curve that BW Com ( $P=0.73$ days) was an anomalous Cepheid, but the result was not conclusive. In a recent paper, Alcock et al. (1999) even argued on evolutionary grounds that stars with periods shorter than about 2.5 days are not classical Cepheids at all. They favored classifying these objects as anomalous Cepheids instead. Although their discussion referred specifically to the LMC, the argument could be applied equally well to the Milky Way Cepheids.

It would be of considerable interest to unambiguously identify the types of individual stars in the intermediateperiod range. For example, nearly all known anomalous Cepheids are outside of the Milky Way and are therefore too faint for detailed observational study. Finding more relatively nearby examples would facilitate studies of their properties and evolutionary history. Similarly, a sample of second-overtone classical Cepheids or overtone type II Cepheids would provide insights into mode selection and other remaining questions surrounding these stars. If some stars in this period range are examples of RR Lyrae with unusual pulsational properties, as was initially suggested for XZ Cet (Simon \& Teays 1982), this could be a key to further understanding of the pulsation and interiors of the RR Lyrae stars. 
Stars in intermediate-period range with periods longer than 1 day have been investigated extensively by, e.g., Diethelm (1990) and Sandage, Diethelm, \& Tammann (1994). In this paper, we will begin an exploration of the population of the lower end of the range. To do this, we present 1116 new photometric observations for 56 stars with periods from 0.6 days to 1 day. These new data, together with published photometry for these and 10 other stars, will be used to consider the possible types of stars that inhabit the intermediate-period range. All of the stars considered in this paper, except BL Boo, are field stars. That star was included because of its possibly unique status as a galactic anomalous Cepheid.

\section{PHOTOMETRY}

\subsection{New Observations}

The observations were made with the CCD photometry system on the Behlen Observatory $0.76 \mathrm{~m}$ telescope. The same methods were used for the observations and reductions as described in previous papers (see Schmidt 1991 and references therein). The new data are listed in Table 1. For most of the stars discussed here, we have also incorporated earlier photometry (see below). The author will provide complete tabulations of all the data upon request.

Most of the stars were previously included in publications resulting from the Behlen Observatory Variable Star Survey (Loomis, Schmidt, \& Simon 1988; Schmidt et al. 1990; Schmidt 1991; Schmidt, Chab, \& Reiswig 1995; Schmidt \& Seth 1996). However, there are nine stars for which new observations are presented here but which were not included

TABLE 1

РноTOMETRIC Data

\begin{tabular}{|c|c|c|c|}
\hline $\begin{array}{c}\text { HJD } \\
(+2,440,000)\end{array}$ & $V$ & $R$ & $V-R$ \\
\hline \multicolumn{4}{|l|}{ AB UMa: } \\
\hline $10,424.874 \ldots$ & 11.090 & 10.723 & 0.365 \\
\hline $10,424.946 \ldots$ & 11.111 & 10.813 & 0.297 \\
\hline $10,425.008 \ldots$ & 10.927 & 10.671 & 0.255 \\
\hline $10,425.884 \ldots$ & 10.911 & 10.611 & 0.298 \\
\hline $10,425.946 \ldots$ & 10.972 & 10.673 & 0.298 \\
\hline $10,426.005 \ldots$ & 11.028 & 10.700 & 0.327 \\
\hline $10,459.752 \ldots$ & 11.054 & 10.785 & 0.266 \\
\hline $10,460.752 \ldots$ & 11.016 & 10.686 & 0.328 \\
\hline $10,460.878 \ldots$ & 11.084 & 10.769 & 0.314 \\
\hline $10,488.692 \ldots$ & 10.741 & 10.502 & 0.239 \\
\hline $10,488.752 \ldots$ & 10.814 & 10.547 & 0.267 \\
\hline $10,501.816 \ldots$ & 10.742 & 10.500 & 0.241 \\
\hline $10,501.946 \ldots$ & 10.801 & 10.552 & 0.249 \\
\hline \multicolumn{4}{|l|}{ FW Peg: } \\
\hline $10,606.857 \ldots$ & 14.288 & 13.904 & 0.384 \\
\hline $10,642.840 \ldots$ & 14.208 & 13.828 & 0.375 \\
\hline $10,655.677 \ldots$ & 14.407 & 14.074 & 0.327 \\
\hline $10,655.708 \ldots$ & 14.389 & 14.028 & 0.357 \\
\hline $10,655.769 \ldots$ & 13.671 & 13.459 & 0.204 \\
\hline $10,656.841 \ldots$ & 14.373 & 13.990 & 0.376 \\
\hline $10,683.774 \ldots$ & 14.309 & 13.949 & 0.358 \\
\hline $10,683.842 \ldots$ & 14.314 & 13.956 & 0.356 \\
\hline
\end{tabular}

NoTE.-Table 1 is available in its entirety in the electronic edition of the Astronomical Journal. A portion is shown here for guidance regarding its form and content. in our previous publications. For completeness, these stars are listed in Table 2, along with the various parameters that were provided for the stars in the earlier publications. Column (1) identifies each star by its variable star name. Columns (2)-(5) give information on the comparison stars. In particular, column (2) gives the number of comparison stars in the field of each star, columns (3) and (4) list the standard errors of the mean comparison star $V$ and $R$ magnitudes, and column (5) gives the number of photometric nights included in the comparison star means. Columns (6) and (7) give the intensity mean magnitudes for the variables. The adopted periods are listed in column (8), while column (9) indicates the estimated uncertainties in the periods in units of the last cited decimal place for periods determined from our photometry. The epoch of maximum is given in column (11). The last two columns list the amplitudes in $V$ and $R$.

The stars discussed in this paper are listed in Table 3, including both those for which new data were obtained and an additional 10 for which only data from the literature were used. The star is identified in the first column, and its approximate period is given in the second column. The third column gives a reference to the source of published data. The total number of light-curve points is listed in the fourth column, where the number in parentheses is the number of new points added in the present study. The remainder of this table is described below.

For about half of the stars, the periods adopted in the papers referenced in column (3) or in the GCVS produce satisfactory light curves. The exceptions are listed in Table 4 , where we give new periods derived from all the available Behlen Observatory data or in two cases, BL Boo and XZ $\mathrm{Cet}$, data from the literature. The estimated uncertainties in the periods are given in units of the last cited decimal place. The last column indicates whether the period was adjusted by less than $1 \%$ (denoted by A) or by more than $1 \%$ (S) from the previous value. In two cases, there was no earlier period (N). The light curves for all of the stars in Table 3 are plotted in Figure 1.

\subsection{Light-Curve Scatter}

Since the discussion below places weight on light-curve stability, we should consider whether the scatter apparent in some of the light curves (those for stars classed as mo and si below) is real. There are two possible sources of spurious scatter, photometric error, and variations in a comparison star.

Photometric errors are generally small compared with the observed scatter. The only star for which there might be a concern on this point is V742 Cyg, the faintest star in our sample. While the formal errors of the photometry are not sufficient to account for the scatter, at faint magnitudes the true uncertainty may be larger. Thus, there is some doubt regarding the reality of the light-curve scatter, which cannot be fully resolved with our existing data. However, since the $V$ and $R$ magnitudes are determined differentially relative to comparison stars on separate CCD frames, their random errors will be uncorrelated. We tested for this by calculating the residuals of the $V$ and $R$ magnitudes from the mean curves. These residuals were found to be correlated with a Pearson correlation coefficient of 0.72 . This is an indication that most of the scatter in the light curve is real. More accurate photometry using the new Behlen Observatory CCD 
TABLE 2

Stars Not INCLUded in EARLier Papers

\begin{tabular}{|c|c|c|c|c|c|c|c|c|c|c|c|c|}
\hline $\begin{array}{l}\text { Star } \\
(1)\end{array}$ & $\begin{array}{l}n_{C} \\
(2)\end{array}$ & $\begin{array}{l}\sigma_{V} \\
(3)\end{array}$ & $\begin{array}{l}\sigma_{R} \\
\text { (4) }\end{array}$ & $\begin{array}{l}n_{n} \\
(5)\end{array}$ & $\begin{array}{c}\langle V\rangle \\
(6)\end{array}$ & $\begin{array}{l}\langle R\rangle \\
(7)\end{array}$ & $\begin{array}{l}\text { Period } \\
\quad(8)\end{array}$ & $\begin{array}{l}\text { Err. }^{\text {a }} \\
(9)\end{array}$ & $\begin{array}{c}\text { Comments } \\
\text { (10) }\end{array}$ & $\begin{array}{l}\text { Epoch } \\
\text { (11) }\end{array}$ & $\begin{array}{l}\Delta V \\
(12)\end{array}$ & $\begin{array}{l}\Delta R \\
(13)\end{array}$ \\
\hline V865 Oph... & 2 & 11 & 12 & 6 & 12.68 & 12.37 & 0.63831 & 1 & $\mathrm{~N}$ & 10529.97 & 0.92 & 0.75 \\
\hline V448 Her ... & 2 & 5 & 7 & 7 & 13.49 & 13.25 & 0.653529 & $\ldots$ & G & 9901.80 & 0.88 & 0.74 \\
\hline BG Eri ........ & 2 & 15 & 10 & 4 & 13.58 & 13.30 & 0.65995 & 4 & A & 10830.58 & 0.60 & 0.44 \\
\hline DV Vir ........ & 1 & 4 & 6 & 3 & 13.83 & 13.58 & 0.66269 & 1 & A & 10607.71 & 0.96 & 0.78 \\
\hline BY Vir ........ & 1 & 14 & 8 & 9 & 14.05 & 13.78 & 0.66733 & 1 & A & 9710.92 & 0.53 & 0.45 \\
\hline ET Hya ....... & 2 & 6 & 7 & 5 & 12.05 & 11.79 & 0.685524 & $\ldots$ & G & 10425.00 & 0.97 & 0.83 \\
\hline AC And ...... & 3 & 7 & 7 & 14 & 10.97 & 10.61 & 0.71124243 & $\ldots$ & $\mathrm{F}$ & 10319.63 & 0.63 & 0.46 \\
\hline V362 Her ... & 1 & 9 & 8 & 10 & 14.39 & 14.13 & 0.718297 & $\ldots$ & G & 9909.77 & 1.22 & 0.94 \\
\hline UY CrB ...... & 2 & 8 & 12 & 5 & 12.74 & 12.51 & 0.92914 & 5 & $\mathrm{~N}$ & 10950.87 & 0.94 & 0.74 \\
\hline
\end{tabular}

a Uncertainties in periods are listed in units of the last digit given for the period.

b A " G" in column (11) indicates that the adopted period comes from the GCVS, while an "A " indicates that the period was adjusted slightly (less than 1\%) using the present photometry. An " $\mathrm{N}$ " means that no period was listed in the GCVS, and the adopted period was determined solely from the present photometry. An "F" refers to the period cited by Fitch \& Szeidl 1976.

TABLE 3

Intermediate-Period Cepheid Strip Stars

\begin{tabular}{|c|c|c|c|c|c|c|c|c|c|c|}
\hline $\begin{array}{c}\text { Star } \\
(1)\end{array}$ & $\begin{array}{l}\text { Period } \\
\text { (2) }\end{array}$ & $\begin{array}{l}\text { Ref. } \\
\text { (3) }\end{array}$ & $\begin{array}{l}N \\
(4)\end{array}$ & $\begin{array}{c}\text { Type } \\
\text { (5) }\end{array}$ & $\begin{array}{l}R_{21} \\
(6)\end{array}$ & $\begin{array}{l}\text { Err. } \\
\text { (7) }\end{array}$ & $\begin{array}{l}R_{31} \\
(8)\end{array}$ & $\begin{array}{l}\text { Err. } \\
(9)\end{array}$ & $\begin{array}{l}\phi_{21} \\
(10)\end{array}$ & $\begin{array}{l}\text { Err. } \\
\text { (11) }\end{array}$ \\
\hline AB UMa.... & 0.600 & c & $28(13)$ & mo: & 0.34 & 0.05 & 0.12 & 0.05 & 4.47 & 0.17 \\
\hline FW Peg....... & 0.600 & c & $41(29)$ & mo & 0.41 & 0.08 & 0.28 & 0.07 & 4.01 & 0.22 \\
\hline KN Lyr....... & 0.601 & d & $24(12)$ & $a b$ & 0.54 & 0.03 & 0.37 & 0.03 & 4.22 & 0.08 \\
\hline AN Per........ & 0.602 & $\mathrm{~b}$ & $69(37)$ & $a b$ & 0.39 & 0.02 & 0.28 & 0.02 & 4.04 & 0.05 \\
\hline WW Leo ..... & 0.603 & c & $19(9)$ & $a b$ & 0.46 & 0.03 & 0.35 & 0.02 & 4.16 & 0.07 \\
\hline DY And ...... & 0.603 & $\mathrm{~b}$ & 49 (33) & $a b$ & 0.37 & 0.03 & 0.29 & 0.03 & 3.71 & 0.11 \\
\hline GH Vir........ & 0.605 & d & $29(14)$ & mo & $\ldots$ & $\ldots$ & $\ldots$ & $\ldots$ & $\ldots$ & $\ldots$ \\
\hline DZ Peg......... & 0.607 & d & $28(16)$ & $\mathrm{ab}$ & 0.57 & 0.03 & 0.37 & 0.03 & 4.05 & 0.07 \\
\hline DU And..... & 0.607 & c & $43(26)$ & $a b$ & 0.44 & 0.03 & 0.35 & 0.03 & 3.82 & 0.07 \\
\hline TU Per ........ & 0.607 & c & $23(10)$ & $a b$ & 0.55 & 0.04 & 0.36 & 0.04 & 3.97 & 0.09 \\
\hline BR Cas........ & 0.608 & $\mathrm{~b}$ & $37(0)$ & $a b$ & 0.46 & 0.02 & 0.25 & 0.01 & 4.29 & 0.04 \\
\hline DT Gem..... & 0.611 & $\mathrm{~b}$ & $58(40)$ & si & 0.19 & 0.05 & 0.14 & 0.05 & 3.63 & 0.26 \\
\hline GT Her ....... & 0.614 & c & $46(33)$ & mo & 0.42 & 0.04 & 0.21 & 0.04 & 4.06 & 0.12 \\
\hline CX Lyr........ & 0.617 & c & $35(23)$ & mo & 0.46 & 0.10 & 0.23 & 0.09 & 3.96 & 0.26 \\
\hline AS Cnc........ & 0.618 & b & $35(13)$ & $a b$ & 0.51 & 0.03 & 0.36 & 0.03 & 3.96 & 0.08 \\
\hline CQ Lac ....... & 0.620 & d & $30(17)$ & $a b$ & 0.51 & 0.03 & 0.33 & 0.02 & 3.89 & 0.06 \\
\hline CW Her ..... & 0.624 & $\mathrm{~b}$ & $29(20)$ & $a b$ & 0.49 & 0.04 & 0.38 & 0.03 & 3.94 & 0.09 \\
\hline RV CMi..... & 0.625 & d & $32(21)$ & $a b$ & 0.49 & 0.03 & 0.37 & 0.03 & 3.90 & 0.08 \\
\hline XZ Lac........ & 0.630 & d & $62(27)$ & mo & 0.40 & 0.10 & 0.18 & 0.10 & 3.97 & 0.31 \\
\hline AE Vir ......... & 0.634 & $\mathrm{c}$ & $33(22)$ & $\mathrm{ab}$ & 0.54 & 0.02 & 0.32 & 0.02 & 4.01 & 0.04 \\
\hline BK UMa.... & 0.636 & $\mathrm{~b}$ & $55(30)$ & $a b$ & 0.36 & 0.03 & 0.14 & 0.03 & 4.21 & 0.10 \\
\hline GO Нya ...... & 0.636 & c & $18(6)$ & $a b$ & 0.45 & 0.05 & 0.25 & 0.05 & 4.11 & 0.14 \\
\hline V865 Oph... & 0.638 & e & $20(20)$ & $a b$ & 0.53 & 0.04 & 0.29 & 0.04 & 4.14 & 0.11 \\
\hline UU UMa ... & 0.645 & c & $23(12)$ & $a b$ & 0.39 & 0.04 & 0.32 & 0.04 & 3.94 & 0.13 \\
\hline IU Cas ........ & 0.649 & $\mathrm{c}$ & $41(29)$ & $a b$ & 0.55 & 0.03 & 0.35 & 0.02 & 3.99 & 0.06 \\
\hline AG Her....... & 0.649 & d & $26(11)$ & mo: & 0.42 & 0.06 & 0.25 & 0.06 & 3.94 & 0.18 \\
\hline V448 Her ... & 0.654 & e & $26(26)$ & $a b$ & 0.50 & 0.02 & 0.32 & 0.02 & 4.14 & 0.04 \\
\hline CM Ori ....... & 0.656 & d & $23(6)$ & $a b$ & 0.51 & 0.05 & 0.30 & 0.04 & 4.08 & 0.11 \\
\hline BG Eri ........ & 0.660 & e & $22(22)$ & mo & $\ldots$ & $\ldots$ & $\ldots$ & $\ldots$ & $\ldots$ & $\ldots$ \\
\hline VY Boo....... & 0.662 & $\mathrm{c}$ & $45(15)$ & $a b$ & 0.48 & 0.02 & 0.37 & 0.02 & 3.86 & 0.05 \\
\hline DV Vir ........ & 0.663 & e & $39(39)$ & $a b$ & 0.53 & 0.02 & 0.32 & 0.02 & 4.18 & 0.04 \\
\hline BY Vir ......... & 0.667 & e & $51(51)$ & $\mathrm{ab}$ & 0.45 & 0.03 & 0.21 & 0.03 & 4.22 & 0.08 \\
\hline TY Cam...... & 0.670 & b & $44(0)$ & mo: & 0.49 & 0.04 & 0.32 & 0.04 & 3.96 & 0.10 \\
\hline BM Vir........ & 0.672 & $\mathrm{c}$ & $43(22)$ & mo & 0.50 & 0.08 & 0.28 & 0.07 & 4.29 & 0.32 \\
\hline SY Psc......... & 0.674 & $\mathrm{~b}$ & $25(8)$ & $\mathrm{ab}$ & 0.43 & 0.03 & 0.33 & 0.04 & 3.89 & 0.10 \\
\hline BD UMa.... & 0.681 & b & $29(15)$ & $a b$ & 0.57 & 0.05 & 0.30 & 0.05 & 4.06 & 0.12 \\
\hline DH Vir........ & 0.682 & c & $17(6)$ & $a b$ & 0.44 & 0.08 & 0.37 & 0.08 & 4.37 & 0.23 \\
\hline NR Lyr ....... & 0.682 & d & $35(18)$ & $\mathrm{ab}$ & 0.42 & 0.03 & 0.34 & 0.03 & 3.80 & 0.08 \\
\hline X LMi......... & 0.684 & c & $17(3)$ & $a b$ & 0.52 & 0.03 & 0.29 & 0.02 & 4.13 & 0.06 \\
\hline ET Нyа ....... & 0.686 & e & $20(20)$ & $a b$ & 0.57 & 0.04 & 0.37 & 0.03 & 4.04 & 0.09 \\
\hline GZ Cep ....... & 0.695 & d & $26(0)$ & $a b$ & 0.54 & 0.02 & 0.33 & 0.02 & 4.18 & 0.05 \\
\hline FM And..... & 0.695 & $\mathrm{c}$ & $33(20)$ & $a b$ & 0.50 & 0.02 & 0.34 & 0.02 & 3.99 & 0.05 \\
\hline UZ CVn...... & 0.698 & $\mathrm{c}$ & $22(9)$ & $a b$ & 0.53 & 0.02 & 0.39 & 0.02 & 3.96 & 0.04 \\
\hline
\end{tabular}


TABLE 3-Continued

\begin{tabular}{|c|c|c|c|c|c|c|c|c|c|c|}
\hline $\begin{array}{c}\text { Star } \\
(1)\end{array}$ & $\begin{array}{c}\text { Period } \\
\text { (2) }\end{array}$ & $\begin{array}{c}\text { Ref. } \\
\text { (3) }\end{array}$ & $\begin{array}{l}N \\
(4)\end{array}$ & $\begin{array}{l}\text { Type } \\
(5)\end{array}$ & $\begin{array}{l}R_{21} \\
(6)\end{array}$ & $\begin{array}{l}\text { Err. } \\
(7)\end{array}$ & $\begin{array}{l}R_{31} \\
(8)\end{array}$ & $\begin{array}{c}\text { Err. } \\
(9)\end{array}$ & $\begin{array}{c}\phi_{21} \\
(10)\end{array}$ & $\begin{array}{l}\text { Err. } \\
(11)\end{array}$ \\
\hline V768 Oph... & 0.702 & $\mathrm{~b}$ & $40(9)$ & mo & 0.56 & 0.07 & 0.48 & 0.07 & 3.85 & 0.17 \\
\hline EN Lyr........ & 0.703 & d & $41(28)$ & mo & 0.38 & 0.11 & 0.30 & 0.11 & 4.27 & 0.35 \\
\hline GM And .... & 0.707 & $\mathrm{~b}$ & $36(0)$ & $a b$ & 0.37 & 0.03 & 0.36 & 0.03 & 3.98 & 0.11 \\
\hline AC And ..... & 0.711 & e & $70(70)$ & si & 0.08 & 0.09 & 0.12 & 0.10 & 3.20 & 1.20 \\
\hline V716 Her ... & 0.714 & d & $23(9)$ & $a b$ & 0.43 & 0.05 & 0.39 & 0.05 & 3.90 & 0.13 \\
\hline V362 Her ... & 0.718 & e & $23(23)$ & $a b$ & 0.51 & 0.05 & 0.36 & 0.05 & 4.27 & 0.13 \\
\hline AX Leo ....... & 0.727 & $\mathrm{~b}$ & $34(5)$ & mo & 0.42 & 0.03 & 0.21 & 0.03 & 4.16 & 0.09 \\
\hline BW Com.... & 0.734 & $\mathrm{~b}$ & $146(59)$ & mo & 0.30 & 0.04 & 0.15 & 0.03 & 4.09 & 0.13 \\
\hline V692 Her ... & 0.737 & $\mathrm{~b}$ & $39(0)$ & $a b$ & 0.52 & 0.04 & 0.31 & 0.03 & 4.38 & 0.09 \\
\hline UZ Com..... & 0.737 & $\mathrm{~b}$ & $37(0)$ & mo & 0.49 & 0.05 & 0.32 & 0.05 & 4.20 & 0.14 \\
\hline CF Leo....... & 0.739 & $\mathrm{c}$ & $29(6)$ & $\mathrm{ab}$ & 0.39 & 0.04 & 0.29 & 0.03 & 3.98 & 0.10 \\
\hline CV Del....... & 0.739 & $\mathrm{~b}$ & $43(6)$ & $a b$ & 0.48 & 0.03 & 0.22 & 0.03 & 4.48 & 0.08 \\
\hline SX Tri........ & 0.743 & $\mathrm{~b}$ & $33(8)$ & $a b$ & 0.34 & 0.03 & 0.22 & 0.03 & 4.48 & 0.09 \\
\hline V599 Her ... & 0.751 & $\mathrm{~b}$ & $36(15)$ & mo: & 0.38 & 0.05 & 0.28 & 0.05 & 4.60 & 0.14 \\
\hline OX Her...... & 0.757 & $\mathrm{~b}$ & $48(5)$ & $a b$ & 0.42 & 0.02 & 0.25 & 0.02 & 4.29 & 0.07 \\
\hline CY Com..... & 0.758 & $\mathrm{~b}$ & $40(12)$ & $a b$ & 0.47 & 0.03 & 0.27 & 0.03 & 4.20 & 0.08 \\
\hline V486 Her ... & 0.806 & $\mathrm{a}$ & $72(0)$ & $a b$ & 0.42 & 0.02 & 0.20 & 0.02 & 4.52 & 0.07 \\
\hline BL Boo ...... & 0.821 & $\mathrm{f}$ & $107(0)$ & si & 0.09 & 0.01 & 0.01 & 0.01 & 4.37 & 0.16 \\
\hline XZ Cet ....... & 0.823 & $\mathrm{~g}$ & $313(0)$ & si & 0.16 & 0.01 & 0.10 & 0.01 & 5.12 & 0.05 \\
\hline DR Lyr ...... & 0.831 & $\mathrm{~b}$ & $38(6)$ & $a b$ & 0.44 & 0.05 & 0.21 & 0.04 & 4.56 & 0.13 \\
\hline KP Cyg ...... & 0.856 & $\mathrm{a}$ & $46(0)$ & $a b$ & 0.42 & 0.01 & 0.21 & 0.01 & 4.65 & 0.03 \\
\hline UY CrB ..... & 0.929 & $\mathrm{e}$ & $26(26)$ & $a b$ & 0.48 & 0.02 & 0.25 & 0.02 & 4.60 & 0.06 \\
\hline V742 Cyg ... & 0.936 & $\mathrm{~b}$ & $70(26)$ & mo: & 0.38 & 0.06 & 0.20 & 0.06 & 4.82 & 0.20 \\
\hline
\end{tabular}

a Previous observations from Loomis et al. 1988.

b Previous observations from Schmidt 1991.

c Previous observations from Schmidt et al. 1995.

d Previous observations from Schmidt \& Seth 1996.

e This star has not appeared in previous papers.

f Data were obtained from Zinn \& Dahn 1976, McCarthy \& Nemec 1997, and Corwin, Carney, \& Nifong 1999. BL Boo is the anomalous Cepheid V19 in NGC 5466.

g Data were obtained from Dean et al. 1977 and Teays \& Simon 1985.

will allow confirmation. In the mean time, we will treat the data for V742 Cyg with caution.

When there is more than one comparison star in the CCD field, they were checked for stability against each other. Thus, we only need to be concerned about the comparison stars when there is only one. There were seven such cases out of about 20 stars with noticeable light-curve scatter: FW Peg, GH Vir, AG Her, AX Leo, BW Com, UZ Com, and V599 Her. As discussed below, all of these, except BW Com, are candidates for the Blazhko effect, and we plan further observations to confirm this. To verify the constancy of the comparison star for BW Com, GSC 0144-02206 (denoted by C1 here), another star was located (GSC 01444-02004, denoted by $\mathrm{C} 2$ ) that is close enough on the sky to $\mathrm{C} 1$ (but not to BW Com itself) to fit in our CCD field and serve as a check star. Frames were taken containing both $\mathrm{C} 1$ and $\mathrm{C} 2$ on 28 nights. We searched for correlations among the differential colors and magnitudes and for periodicities in them that would indicate variation in $\mathrm{C} 1$ rather than BW Com. All of these tests confirmed the stability of $\mathrm{Cl}$, and we conclude that the light-curve scatter is intrinsic to BW Com.

\section{LIGHT-CURVE PROPERTIES}

\subsection{Morphology}

We examined the light curves visually and divided them into three groups according to appearance. The majority of the light curves exhibit the rapid rise to maximum light and slow decline to minimum typical of Bailey type $a b$ and show little scatter about a mean light curve; these are identified by the letters ab in column (5) of Table 3. Stars that exhibit the ab light-curve shape but have considerable scatter in their light curves (possibly suggestive of modulation by the Blazhko effect) are identified by mo in column (5). The distinction between $\mathrm{ab}$ and mo is somewhat arbitrary, in that there is a continuous range in the degree of scatter and that it is affected by the accuracy of the observations. In principle, we could distinguish scatter due to random errors from true light-curve modulation by examining the consistency of observations from the same night. In practice, this was not very helpful given the paucity of multiple observations on a single night at crucial phases. In every case, this test supported the classifications in Table 3 but generally was not decisive. Hence, some of the stars are labeled "mo:" to denote uncertainty as to whether they are $a b$ or mo. We have identified nearly sinusoidal light curves, which often show significant scatter by si. Placing a star in this class relied mainly on minimum light occurring near phase 0.5 .

We were tempted to subdivide the ab stars further in as much as a few exhibit shoulders or inflections during rising light. Examples of the former include V692 $\operatorname{Her}(P=0.737$ days), SX Tri $(P=0.743$ days $)$, and DR And $(P=0.831$ days), while GO Hya $(P=0.636$ days $)$ and $C V$ Del $(P=0.739$ days $)$ exemplify the latter. However, these deviations in morphology seem minor compared with the distinc- 
TABLE 4

Revised Periods

\begin{tabular}{|c|c|c|c|}
\hline Star & New Period & Err. & Comments \\
\hline FW Peg ....... & 0.60049 & 2 & A \\
\hline AN Per........ & 0.602086 & 3 & A \\
\hline DY And ...... & 0.603087 & 5 & A \\
\hline DU And..... & 0.606842 & 2 & A \\
\hline TU Per ........ & 0.607067 & 4 & A \\
\hline DZ Peg........ & 0.607351 & 4 & A \\
\hline GT Her ....... & 0.613745 & 15 & A \\
\hline AS Cnc........ & 0.617531 & 6 & A \\
\hline CQ Lac ....... & 0.620047 & 3 & A \\
\hline RV CMi ..... & 0.625450 & 5 & A \\
\hline AE Vir ........ & 0.633864 & 4 & A \\
\hline BK UMa.... & 0.635666 & 4 & A \\
\hline V865 Oph... & 0.63831 & 1 & $\mathrm{~N}$ \\
\hline UU UMa ... & 0.644833 & 5 & A \\
\hline IU Cas ........ & 0.649418 & 4 & A \\
\hline BG Eri ........ & 0.65995 & 4 & A \\
\hline VY Boo....... & 0.661716 & 3 & A \\
\hline DV Vir ........ & 0.66269 & 1 & A \\
\hline BY Vir ........ & 0.66733 & 1 & $\mathrm{~S}$ \\
\hline SY Psc......... & 0.673548 & 3 & A \\
\hline BD UMa.... & 0.681134 & 5 & A \\
\hline X LMi.......... & 0.68434 & 2 & A \\
\hline FM And..... & 0.695495 & 5 & A \\
\hline $\mathrm{UZCVn} . . . .$. & 0.697793 & 4 & A \\
\hline AX Leo ........ & 0.726845 & 10 & A \\
\hline V599 Her ... & 0.750605 & 10 & A \\
\hline OX Her ....... & 0.757358 & 4 & A \\
\hline BL Boo ....... & 0.821312 & 2 & A \\
\hline XZ Cet ......... & 0.823126 & 3 & A \\
\hline UY CrB ..... & 0.92914 & 5 & $\mathrm{~N}$ \\
\hline V742 Cyg ... & 0.93632 & 3 & A \\
\hline
\end{tabular}

a An " A" indicates that the period was adjusted slightly (less than $1 \%$ ) from that adopted previously or that given in the GCVS, while an "S" indicates that the adopted period differs significantly from the previous value (more than $1 \%$ ). An " $\mathrm{N}$ " means that no period was listed in the GCVS, and the adopted period was determined solely from the present photometry.

tion between the $\mathrm{ab}$ and the si stars. Additionally, they are not manifest in the Fourier parameters discussed below.

\subsection{Fourier Decomposition}

Fourier decomposition was performed on the light curves as described in Schmidt et al (1990). Several of the Fourier parameters are listed in columns (6), (8), and (10) of Table 3 , while the corresponding standard deviations of these quantities are listed in columns (7), (9), and (11). The latter were calculated with the formulas of Petersen (1986). Fifth- or sixth-order Fourier series fit the data very well for nearly all of the stars. Because of the scatter, stars with mo light curves present difficulties in fitting the Fourier series. However, for all except two of them, GH Vir and BG Eri, there were enough data points to define a reasonable mean light curve, as represented by the tabulated coefficients. Because of the large scatter in the light curve of AC And, higher order fits produced large excursions. Thus, a third-order fit was adopted.
In Figure 2, we have plotted the Fourier parameters from Table 3, together with values from Simon \& Teays (1982), for 20 other Bailey type ab stars. The circles and triangles represent the type ab stars from Table 3 and from Simon \& Teays, respectively. The crosses represent stars with mo light curves, while the plus signs represent the si light curves. Error bars are shown for the stars from Table 3. Stars that fall well outside of the main distribution of points are identified for reference in the discussion below.

It can be seen that the ab stars occupy a well-defined region in each of the diagrams. The only ab star that falls significantly outside of that region is BK UMa in Figure $2 b$. An examination of the light curve of this star in Figure 1 shows that, with a more rounded maximum, it does indeed present a somewhat different morphology than other $a b$ stars. However, we have chosen to leave it in that class, since it does show a rapid rise toward maximum and minimum is near phase 0.75 .

Most of the Fourier coefficients of the mo stars (crosses in the plots) probably fall within the ab region when the errors are taken into account. We conclude that these stars have an underlying ab light curve. This is supported by an examination of the light curves in Figure 1.

Stars with light curves classified as "si" (plus signs) fall significantly below the others of similar period in the $R_{12}$ and $R_{13}$ diagrams. This simply reinforces the visual classification of the light curves as sinusoidal. Figure $2 c$, on the other hand, shows that $\phi_{21}$ is not useful for distinguishing the different types of light curves among intermediateperiod stars.

\subsection{Light-Curve Stability}

An inspection of Figure 1 shows that the large majority of the ab light curves in our sample repeat very well over a number of cycles. However, those in the other two categories exhibit scatter in excess of the errors. In RR Lyrae stars exhibiting the Blazhko effect, both the magnitude at maximum (and sometimes at other phases) and the time of maximum relative to a constant period generally vary over the Blazhko cycle (Tsesevich 1975). Both effects can give rise to scatter in folded light curves, and it is hard to distinguish between them without inordinate amounts of data. Thus, we will characterize the scatter under both assumptions in anticipation of the possibility that stars will be found that exhibit only one of the two variations.

There is reason to believe that light-curve scatter may, in fact, be a consequence of the interior structure of a star and may thus be a valid diagnostic of stellar type. In highly evolved, low-mass stars, the mass is strongly concentrated in the core. The extended envelope has a small density gradient, which leads to strongly nonadiabatic and nonlinear pulsation. As a result, the light curve does not repeat well from cycle to cycle, and there is increased scatter in folded light curves. Thus, the finding of Balasz-Detre \& Detre (1965), that scatter in the times of maximum light were significantly larger for type II Cepheids than for classical Cepheids, non-Blazhko RR Lyrae stars, and other Cepheid strip stars, can be understood in terms of basic stellar properties.

To characterize the scatter in the light curves in a quantitative manner, we will consider the 10 stars listed in Table 5 that have adequate temporal coverage. 

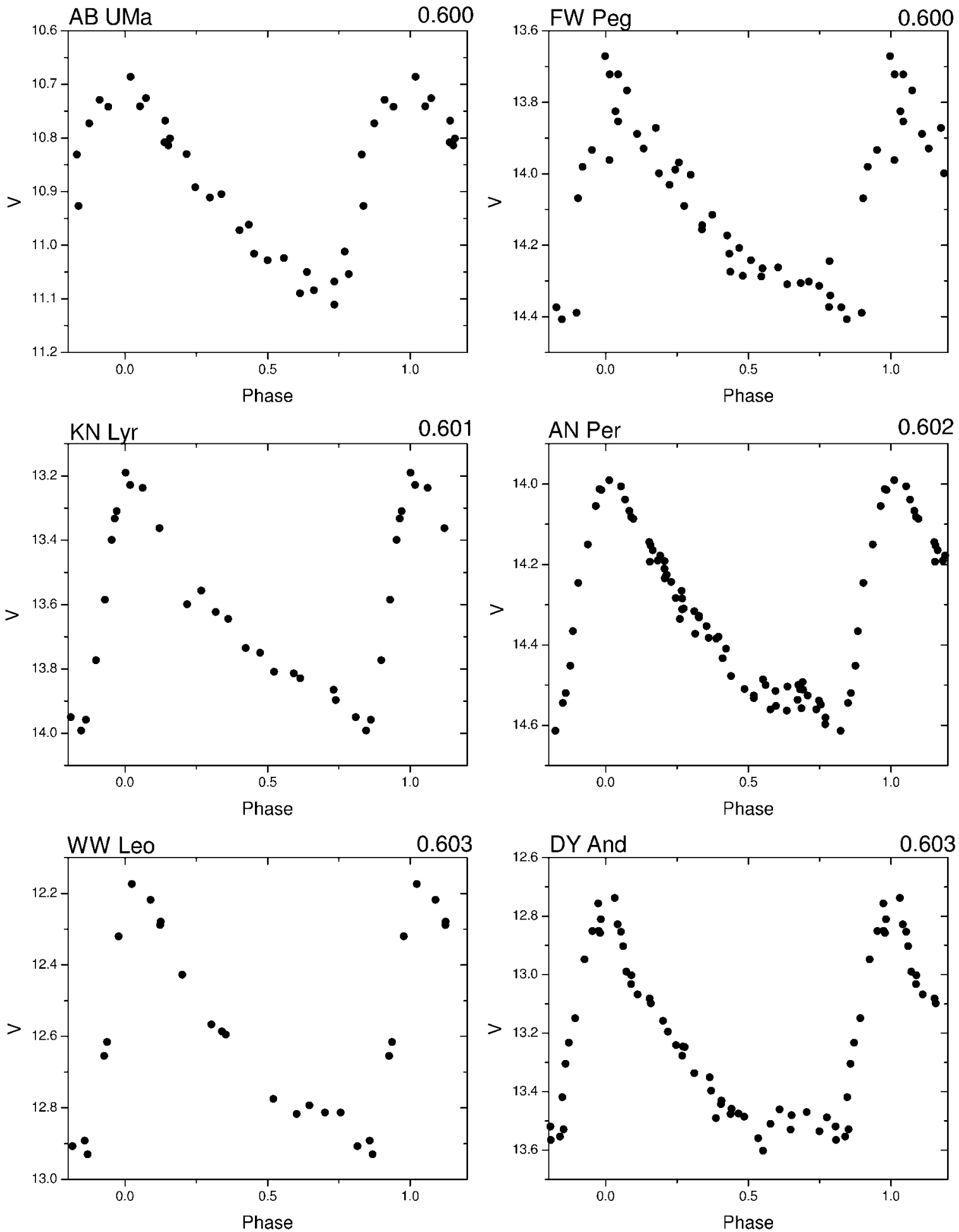

FIG. 1. $-V$ light curves for all the stars from Table 3

We begin by fitting a smooth mean curve to all the photometric data for each star using the cubic B-spline method described by Akerlof et al. (1994). The simplest way to quantify the scatter is to calculate the rms deviations of individual points from the mean light curve. This essentially assumes that the periods or phases are stable and that the scatter is in the brightness alone. Values of the rms scatter,
$\sigma_{V}$, are given in the third column of Table 5. Naturally, the scatter is smaller for the ab stars than for the mo stars, while the si stars exhibit varying degrees of scatter.

Alternatively, if we assume that the scatter is produced entirely by phase shifts, we can follow Balazs-Detre \& Detre (1965) in using the rms scatter of the phase of maximum light around a mean value (denoted here by $\sigma_{\phi}$ ). To obtain 

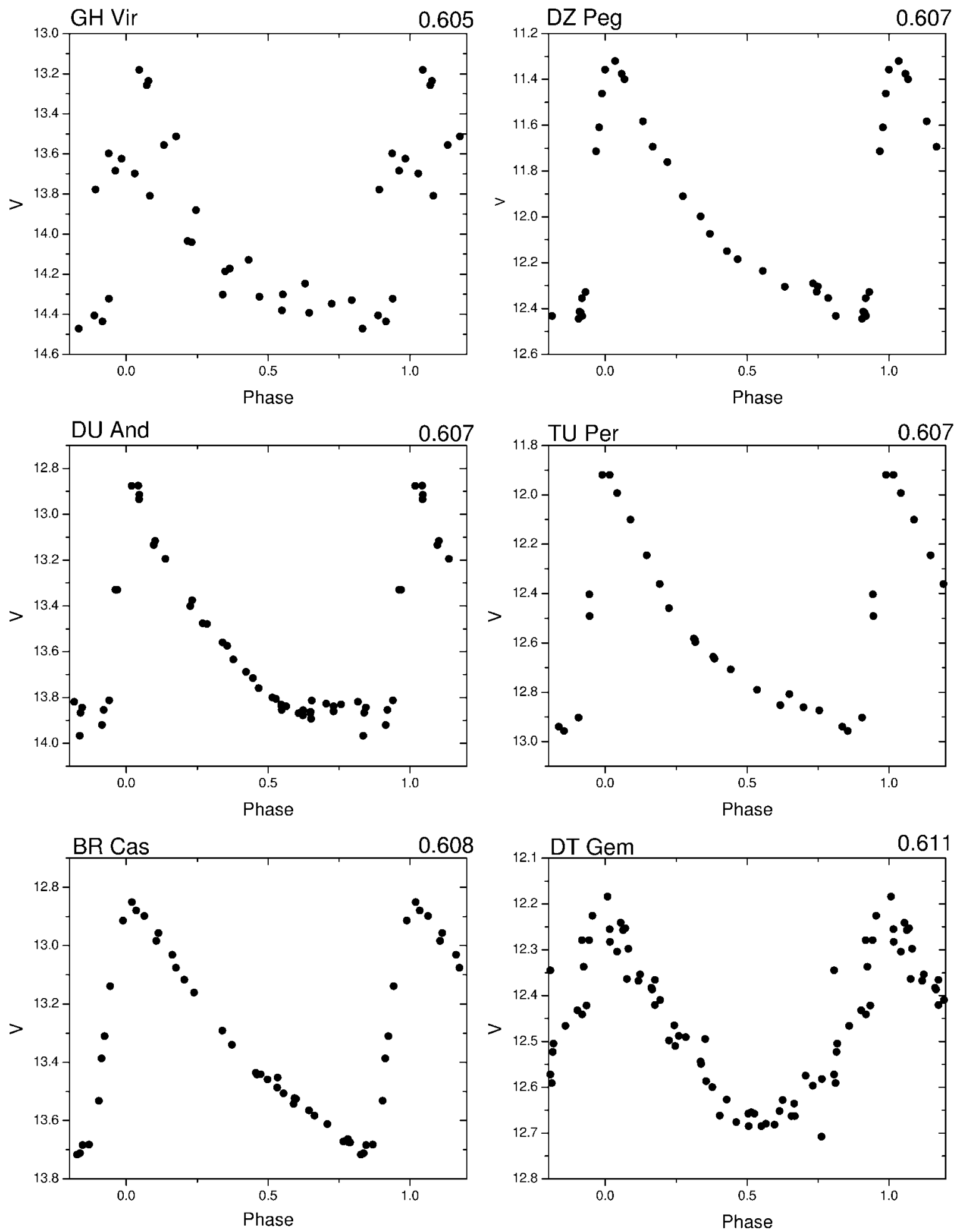

FIG. 1.-Continued

the epochs of maximum, we have used the Hertzsprung method (Hertzsprung 1919), in which the data points from a restricted time interval are shifted in phase to minimize the rms deviations from the mean curve. We only used intervals containing five or more points that were well distributed in phase. The results are contained in column (4) of Table 5, while column (5) lists the number of maxima included in the calculation of $\sigma_{\phi}$. The results are similar to what was found with $\sigma_{V}$.

Note that scatter in the magnitudes will produce scatter in the fitted phase of maximum light, depending on the distribution of the points around the cycle. Similarly, phase 

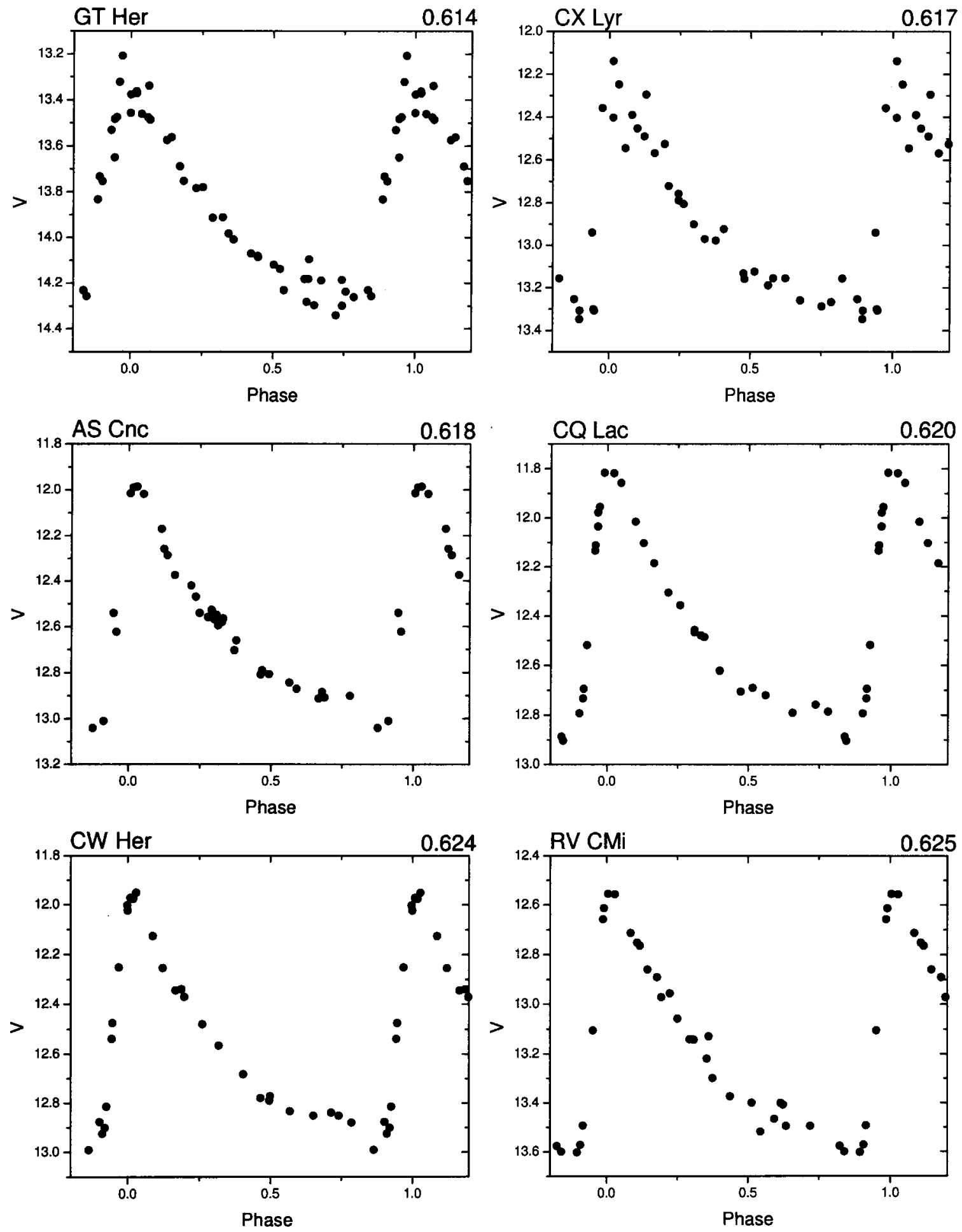

FIG. 1.-Continued

shifts will contribute to the scatter, which is calculated in the magnitudes. Thus, in practice, $\sigma_{V}$ and $\sigma_{\phi}$ are not completely independent and should be considered together.

We have attempted to find periodic behavior in the scatter in the light curves of the si and mo stars listed in Table 5 (except AC And, for which Fitch \& Szeidl 1976 demonstrated multiperiodic behavior). In each case, the mean light curves were subtracted from the measured $V$ magnitudes, and the discrete data-corrected Fourier method (Ferraz-Mello 1981) was used to search for periodicities in the residuals. The most significant period found each case was of marginal significance and accounted for no more than about half of the variance in the residuals. We conclude that the scatter in the light curves of these stars is not associated with a second periodicity of any significance. 

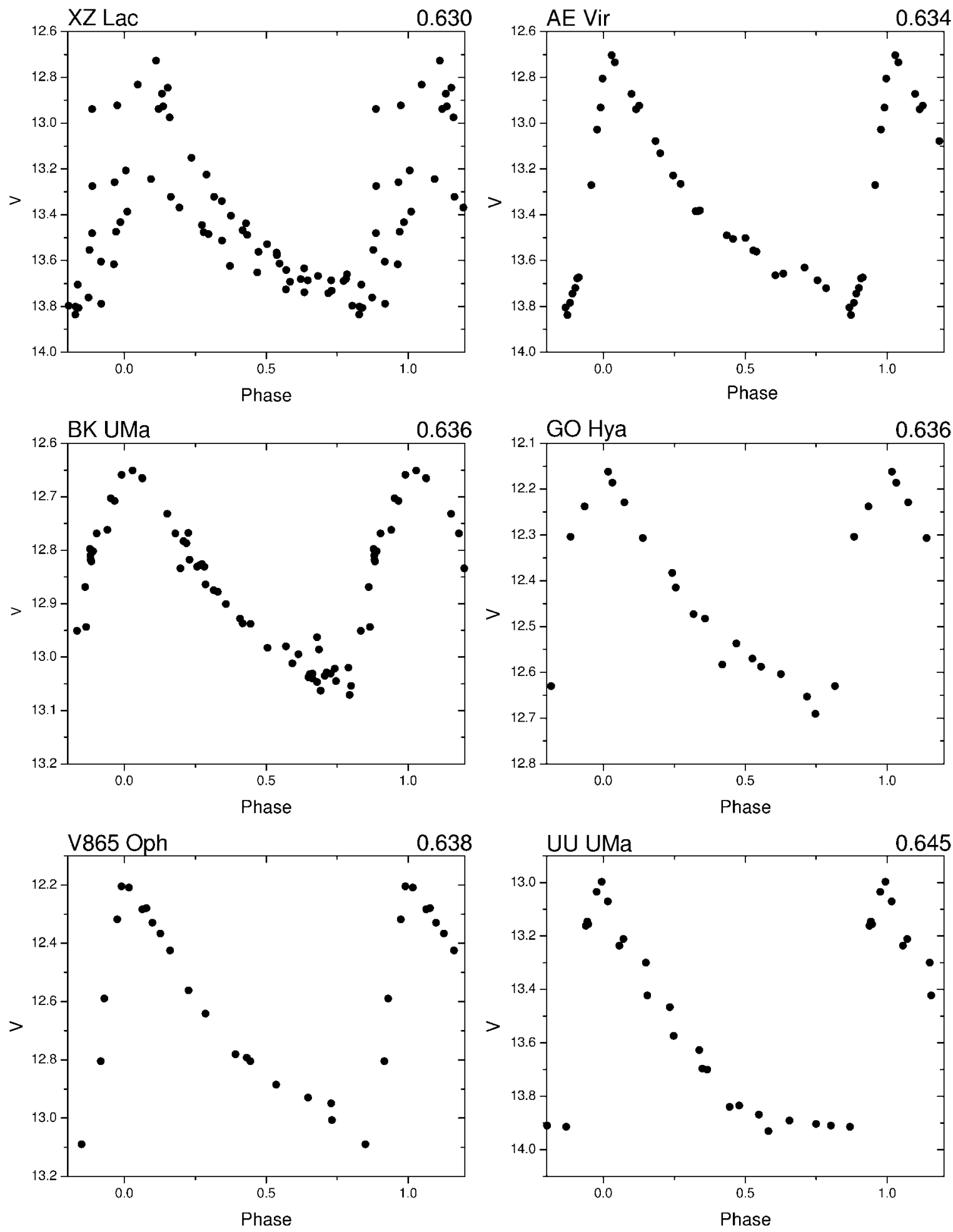

FIG. 1.-Continued

\section{DISCUSSION}

Based on the previous discussion, we will now tentatively separate the stars into groups according to the light curves. We will indicate the likely classification associated with each group, but further investigation is needed to verify them.
The classifications that are proposed are listed in Table 6, along with the various diagnostics that were used.

Clearly, the majority of the stars are non-Blazhko Bailey type ab RR Lyrae stars. In addition to the morphology of the light curve, these stars are recognized by their location in the Fourier diagrams and by their stable light curves, as 

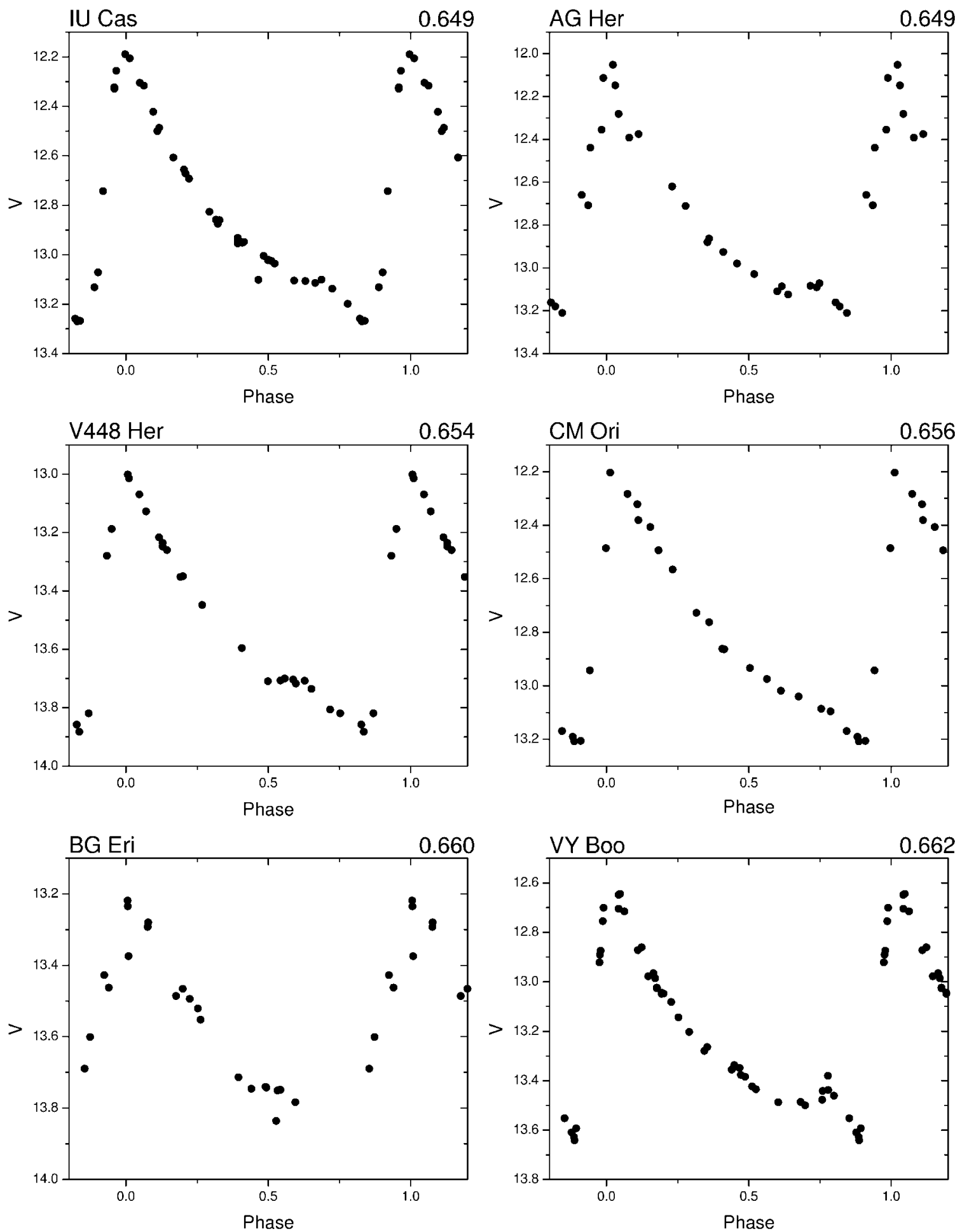

FIG. 1.-Continued

indicated by $\sigma_{V}$ and $\sigma_{\phi}$. In our sample, the number of these stars rapidly decreases from 33 in the range from 0.6 to 0.7 days to three between 0.8 and 0.9 days and to one with a longer period. This is just the long-period tail of the well-known period distribution for RR Lyrae stars.

The stars that we have designated as mo show an ab light curve but lack the stability of the ab group.
Although we do not have sufficient data to study the nature of the modulation of the light curves, it seems likely that most of these stars are affected by the Blazhko effect. In light of a variety of selection effects, our stars can probably not be considered to constitute a randomly drawn sample. Even so, it is worth noting that we may have found 10-15 Blazhko stars (omitting two stars dis- 

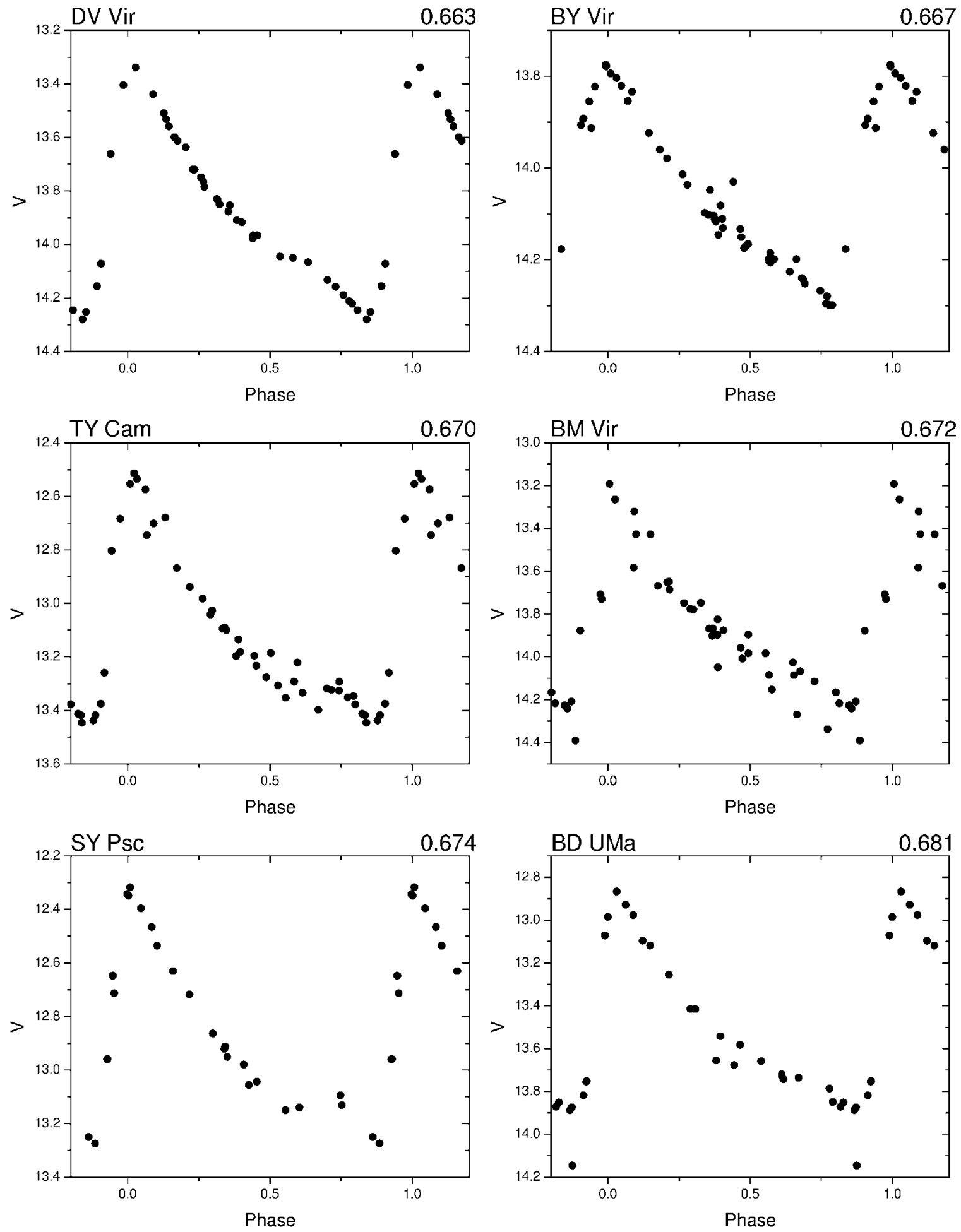

FIG. 1.-Continued

cussed below) out of about 60 presumed RR Lyrae stars. This constitutes $17 \%-25 \%$ of the RR Lyrae stars, which is comparable to the proportion among shorter period RR Lyrae stars (Smith 1995).

In his compilation of Blazhko stars, Smith lists only four with periods longer than 0.6 days, none in common with our program. Furthermore, the GCVS contains no indication that any of these stars exhibit the Blazhko effect, although five are flagged as having variable periods. Thus, we have potentially increased the number and the proportion of these stars at long period significantly. Further intensive observations are planned to verify the presence of the 

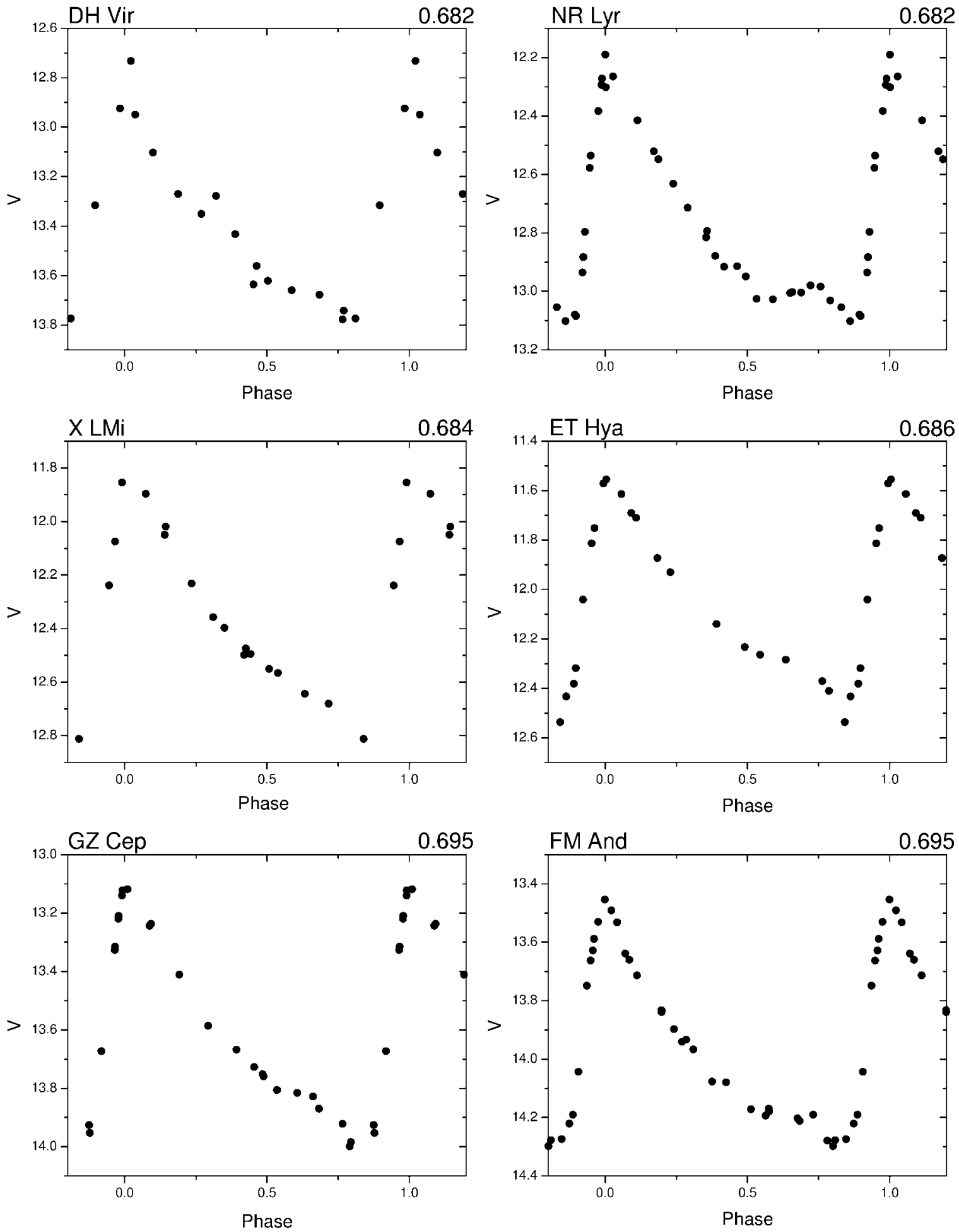

FIG. 1.-Continued

Blazhko effect and to determine the Blazhko periods. In the meantime, this result suggests that the prevalence of the Blazhko effect may not be as dependent on period as some previous studies have indicated (see, e.g., Gloria 1990).

Turning to the si stars, we will not discuss AC And, since its status as a multimode pulsator is well established (Fitch
\& Szeidl 1976; Cox, King, \& Hodson 1978). Similarly, the status of BL Boo as an anomalous Cepheid is secure given that its luminosity is established by its cluster membership (Zinn \& Dahn 1976; Zinn \& King 1982). We note that XZ Cet shares a similar position in the Fourier diagrams with BL Boo and exhibits a similar degree of light-curve stability. 

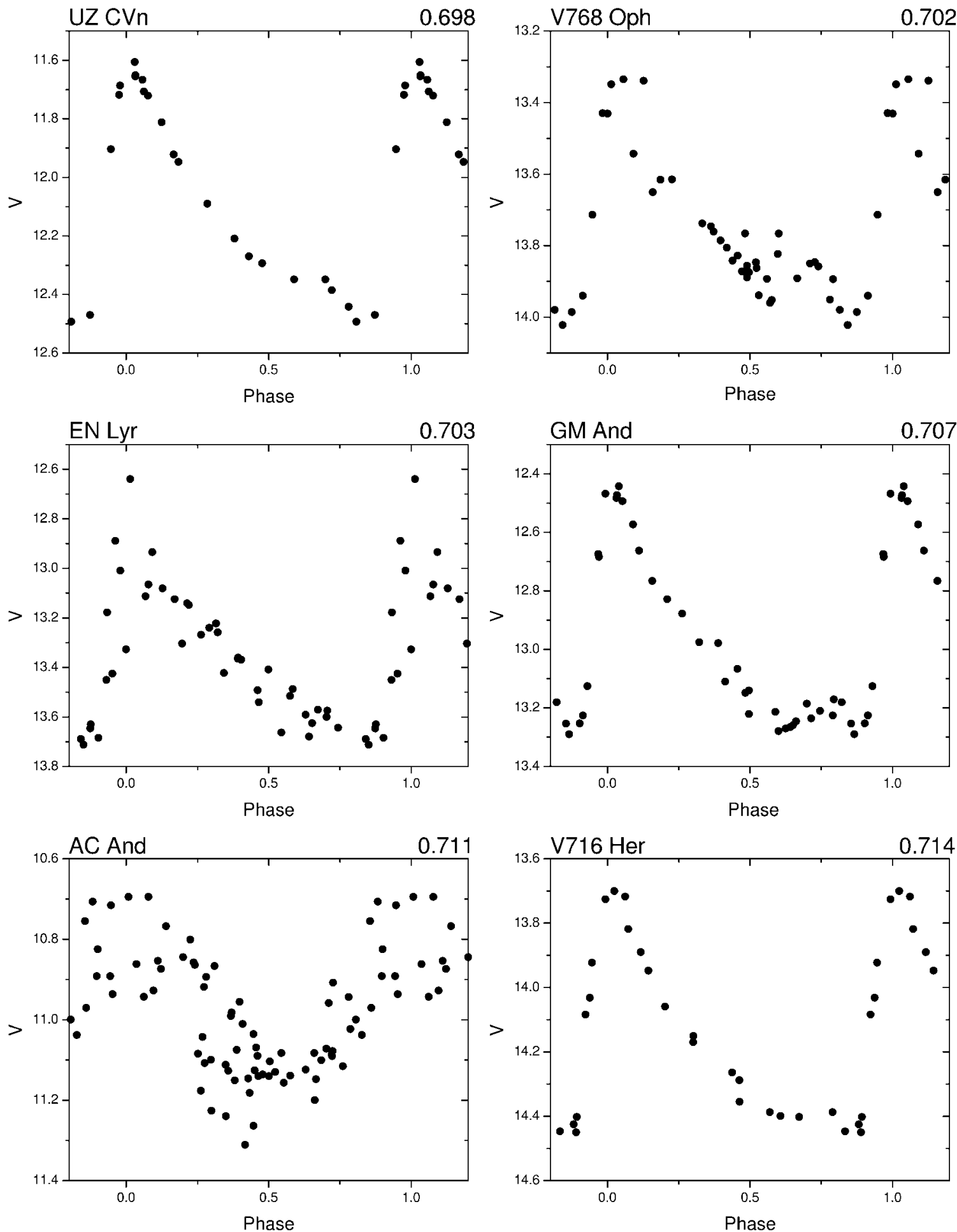

FIG. 1.-Continued

Thus, we tentatively place these two stars in the same category and regard XZ Cet as an anomalous Cepheid.

We note that DT Gem and BW Com share several similarities. Although the light curve of DG Gem is more sinusoidal, both have low values for $R_{31}$ compared with stars of similar period. They both have larger values for both $\sigma_{V}$ and $\sigma_{\phi}$ than the ab stars (Table 5). In fact, the value of $\sigma_{\phi}$ is typi- cal of that found by Balazs-Detre \& Detre (1965) for type II Cepheids. On this basis, we suggest that these two stars should be classed together and may be overtone type II Cepheids, as was suggested for XZ Cet by Teays \& Simon (1985). Although several papers have studied the behavior of the Fourier parameters for type II Cepheids (Petersen \& Diethelm 1986; Simon 1986; Fernie \& Ehlers 1999), they 

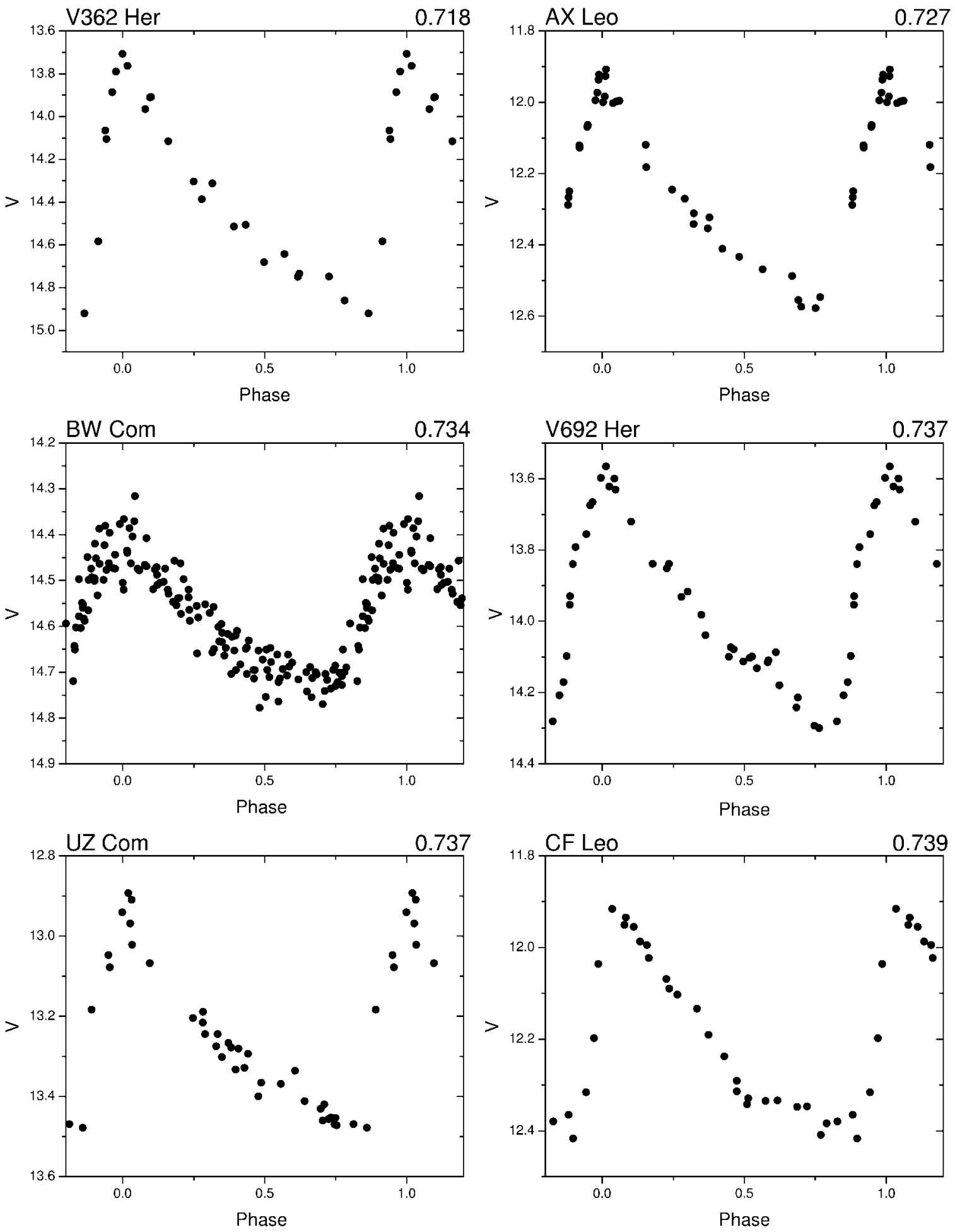

FIg. 1.—Continued

only included stars with periods greater than 1.1 days, which presumably are fundamental-mode oscillators. Thus, they provide no additional insight into the status of DT Gem and BW Com.

Finally, we consider the two longest period stars in our sample, UY CrB and V742 Cyg. Although they have sim- ilar light-curve shapes and the Fourier parameters are similar, an inspection of the light curves shows that the scatter for V742 Cyg is considerably larger than for UY $\mathrm{CrB}$. Unfortunately, as discussed above, we cannot conclude definitely that the scatter in the photometry of V742 Cyg is real. In light of this, we suggest that V742 

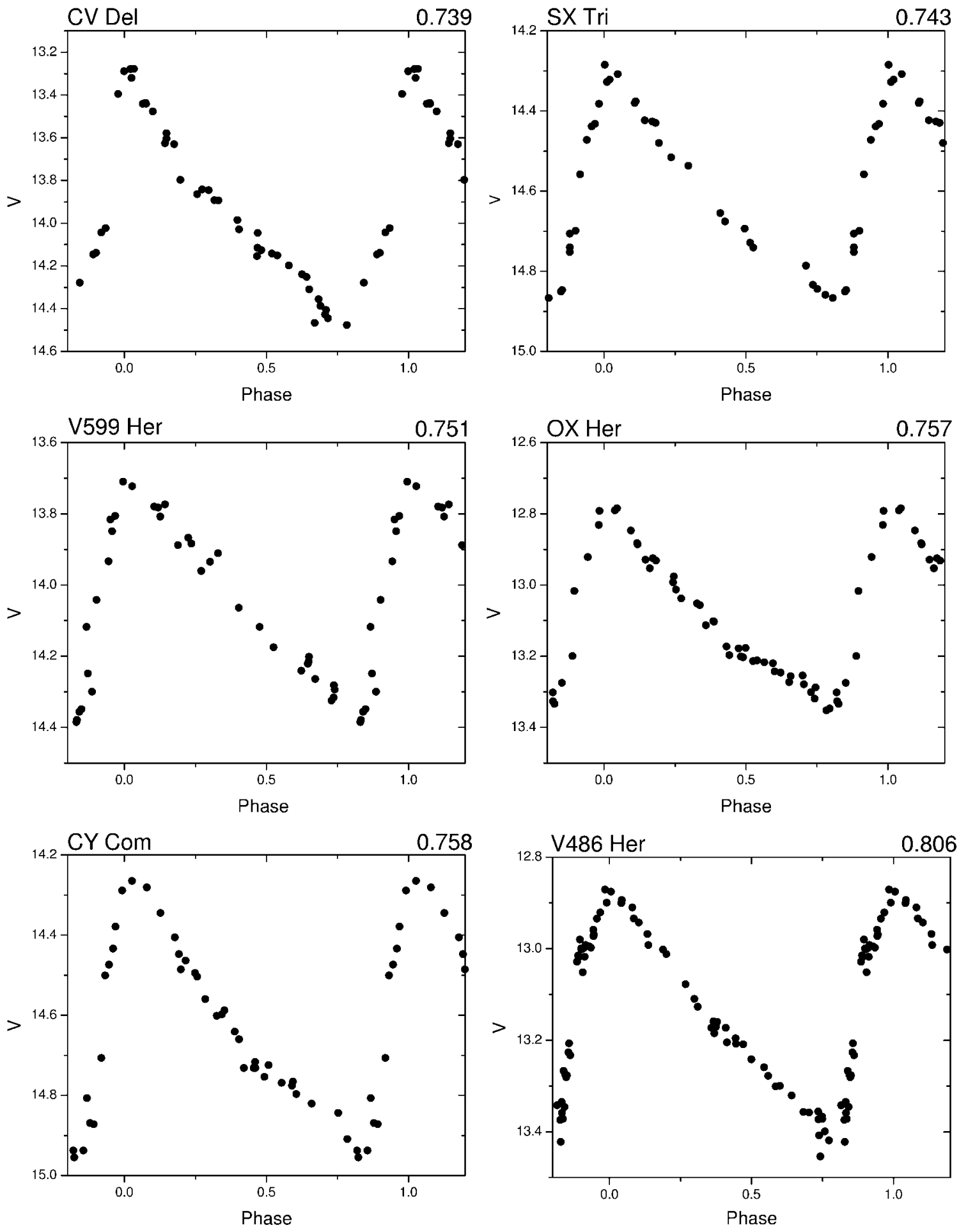

FIG. 1.-Continued

Cyg should be tentatively grouped with DT Gem and BW Com, but a final conclusion must await further photometry. On the other hand, there is no reason to consider UY $\mathrm{CrB}$ to be anything other than a type ab RR Lyrae star.

The above discussion is clearly not definitive, and further observations are needed to confirm the status of many of the stars. Additionally, we have not considered whether some of the longer period stars with ab light curves, especially UY CrB, should be associated with Diethelm's (1990) AHB1 class. Further observations are also needed to address this question and the relationship of the RR Lyrae to the AHB1 stars. Further photometry is planned at Behlen Observatory to further clarify these questions. Spectro- 

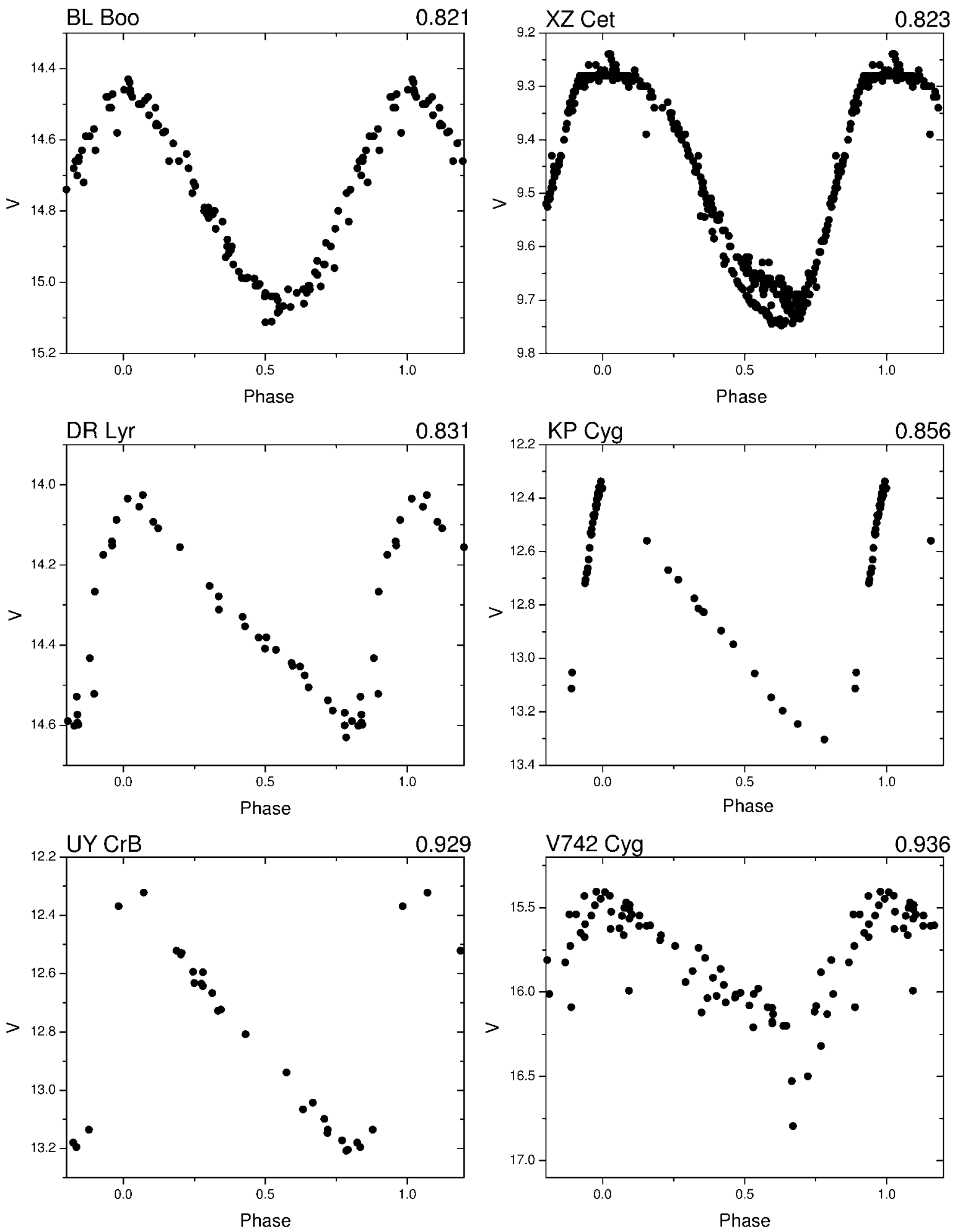

FIg. 1.-Continued

scopic observations are also planned for some stars in the present sample.

This work was supported by the National Science Foundation (NSF) through grants AST 88-15806 and AST 00-
97353. The equipment used was funded through NSF grant AST 85-04072. Some of the analysis was carried out using the facilities of the Minnich Astronomical Computing Center, which was established through a donation by Commander Charles B. Minnich. 

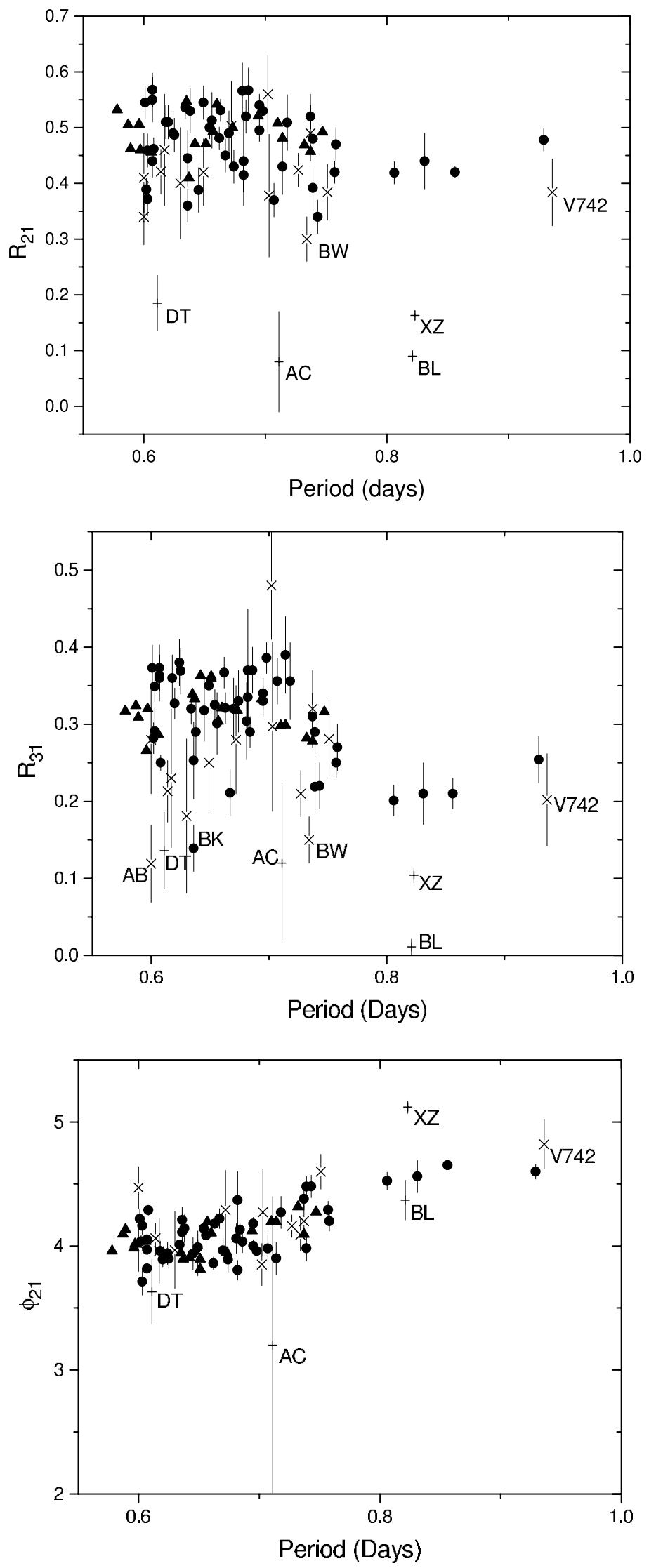

FIG. 2.-Plots for several of the Fourier parameters vs. period. The symbols have the following meaning: triangles denote stars from Simon \& Teays (1982), excluding XZ Cet (shown with a plus sign); circles denote stars from Table 3 with ab light curves; plus signs denote stars with si light curves; and crosses denote stars with mo light curves. Error bars from Table 3 are shown. Individual stars that fall outside of the main distributions are identified by letters as follows: "AB" refers to AB Uma, "AC" to AC And, "BK" to BK Boo, "BL" to BL Boo (=NGC 5466, V19), "BW" to BW Com, "DT" to DT Gem, "XZ" to XZ Cet, and "V742" to V742 Cyg. 
TABLE 5

Light-Curve Stability

\begin{tabular}{ccccc}
\hline \hline $\begin{array}{c}\text { Star } \\
(1)\end{array}$ & $\begin{array}{c}\text { Period } \\
(2)\end{array}$ & $\begin{array}{c}\sigma_{V} \\
(3)\end{array}$ & $\begin{array}{c}\sigma_{\phi} \\
(4)\end{array}$ & $\begin{array}{c}n \\
(5)\end{array}$ \\
\hline ab light curve: & & & & \\
AN Per......... & 0.602 & 0.022 & 0.008 & 7 \\
VY Boo ........ & 0.662 & 0.024 & 0.005 & 5 \\
BY Vir......... & 0.667 & 0.028 & 0.009 & 6 \\
V486 Her...... & 0.806 & 0.021 & 0.003 & 6 \\
mo light curve: & & & & \\
BW Com ....... & 0.734 & 0.040 & 0.021 & 6 \\
V742 Cyg...... & 0.936 & $0.129:$ & 0.014 & 6 \\
si light curve: & & & & \\
DT Gem....... & 0.611 & 0.047 & 0.018 & 7 \\
AC And ........ & 0.711 & 0.093 & 0.032 & 4 \\
BL Boo......... & 0.821 & 0.031 & 0.017 & 9 \\
XZ Cet .......... & 0.823 & 0.021 & 0.006 & 6 \\
\hline
\end{tabular}

TABLE 6

Classification of Stars

\begin{tabular}{cccccc}
\hline \hline Suggested Class & LC Morphology & Fourier Coefficients ${ }^{\mathrm{a}}$ & $\sigma_{V}$ & $\sigma_{\phi}$ & Examples \\
\hline ab RR Lyrae .................... & $a b$ & $\mathrm{~A}$ & $<0.03$ & $<0.01$ & All ab stars \\
Blazhko Effect R R Lyrae .... & mo & A & $\ldots$ & $\ldots$ & Most mo stars \\
Multimode RR Lyrae ......... & si & B & $\sim 0.09$ & $\sim 0.03$ & AC And \\
Anomalous Cepheid ......... & si or mo & C & $<0.03$ & $<0.02$ & BL Boo, XZ Cet \\
Overtone Type II Cepheid ... & si & C & $0.04-0.13$ & $0.018-0.059$ & DT Gem, BW Com, V742 Cyg: \\
\hline
\end{tabular}

a $\mathrm{An}$ " $\mathrm{A}$ " indicates that the coefficients are all in the main group, a " $\mathrm{B}$ " indicates that $R_{21}, R_{31}$, and $\phi_{21}$ are all low, and a " $\mathrm{C}$ " indicates that $R_{21}$ and $R_{31}$ are low, but $\phi_{21}$ is in the main group.

Akerlof, C., et al. 1994, ApJ, 436, 787

Alcock, C., et al. 1999, AJ, 117, 920

. 1995, AJ, 109, 1653

Balazs-Detre, J., \& Detre, L. 1965, in The Position of Variable Stars in the Hertzsprung-Russell Diagram, Veroff. der Remeis-Sternwarte Bamberg IV, No. 40, 184

Corwin, T. M., Carney, B. W., \& Nifong, B. G. 1999, AJ, 118, 2875

Cox, A. N., King, D. S., \& Hodson, S. W. 1978, ApJ, 224, 607

Dean, J. F., Cousins, A. W. J., Bywater, R. A., \& Warren, P. R. 1977, MmRAS, 83, 69

Diethelm, R. 1990, A\&A, 239, 186

Fernie, J. D., \& Ehlers, P. 1999, AJ, 117, 1563

Ferraz-Mello, S. 1981, AJ, 86, 619

Fitch, W. S., \& Szeidl, B. 1976, ApJ, 203, 616

Gloria, K. A. 1990, PASP, 102, 338

Hertzsprung, E. 1919, Astron. Nachr., 210, 17

Kholopov, P. N. 1985, General Catalogue of Variable Stars, Vols. 1 and 2 (4th ed.; Moscow: Nauka)

. 1987, General Catalogue of Variable Stars, Vol. 3 (4th ed.; Moscow: Nauka)

Kukarkin, B. V. 1975, in Variable Stars and Stellar Evolution, ed. V. Sherwood \& L. Plaut (Dordrecht: Reidel), 511

Layden, A. C., Ritter, L. A., Welch, D. L., \& Webb, T. M. A. 1999, AJ, 117,1313

\section{REFERENCES}

Loomis, C., Schmidt, E. G., \& Simon, N. R. 1988, MNRAS, 235, 1059

McCarthy, J. K., \& Nemec, J. M. 1997, ApJ, 482, 203

Nemec, J. M. 1989, in IAU Colloq. 111, The Use of Pulsating Stars in Fundamental Problems in Astronomy, ed. E. Schmidt (Cambridge: Cambridge Univ. Press), 215

Petersen, J. O. 1986, A\&A, 170, 59

Petersen, J. O., \& Diethelm, R. 1986, A\&A, 156, 337

Sandage, A., Diethelm, R., \& Tammann, G. A. 1994, A\&A, 283, 111

Schmidt, E. G. 1991, AJ, 102, 1766

Schmidt, E. G., Chab, J. R., \& Reiswig, D. E. 1995, AJ, 109, 1239

Schmidt, E. G., Loomis, C. G., Groebner, A. T., \& Potter, C. T. 1990, ApJ, 360,604

Schmidt, E. G., \& Seth, A. 1996, AJ, 112, 2769

Simon, N. R. 1986, ApJ, 311, 305

Simon, N. R., \& Teays, T. J. 1982, ApJ, 261, 586

Smith, H. 1995, RR Lyrae Stars (Cambridge: Cambridge Univ. Press)

Teays, T. J., \& Simon, R. R. 1985, ApJ, 290, 683

Tsesevich, V. P. 1975, in Pulsating Stars, ed. B. V. Kukarkin (New York: Wiley), 144

Zinn, R., \& Dahn, C. C. 1976, AJ, 81, 527

Zinn, R., \& King, C. R. 1982, ApJ, 262, 700 